Ion Beam Induced Surface Modulations from Nano to Pico: Optimizing Deposition During Erosion and Erosion During Deposition.

W. J. MoberlyChan, R. Schalek

November 12, 2007

Materials Research Society

Boston, MA, United States

November 24, 2006 through November 30, 2006 
This document was prepared as an account of work sponsored by an agency of the United States government. Neither the United States government nor Lawrence Livermore National Security, LLC, nor any of their employees makes any warranty, expressed or implied, or assumes any legal liability or responsibility for the accuracy, completeness, or usefulness of any information, apparatus, product, or process disclosed, or represents that its use would not infringe privately owned rights. Reference herein to any specific commercial product, process, or service by trade name, trademark, manufacturer, or otherwise does not necessarily constitute or imply its endorsement, recommendation, or favoring by the United States government or Lawrence Livermore National Security, LLC. The views and opinions of authors expressed herein do not necessarily state or reflect those of the United States government or Lawrence Livermore National Security, LLC, and shall not be used for advertising or product endorsement purposes. 


\title{
Ion Beam Induced Surface Modulations from Nano to Pico: Optimizing Deposition During Erosion and Erosion During Deposition.
}

\author{
WARREN J. MOBERLYCHAN* \& RICHARD SCHALEK** \\ *Lawrence Livermore National Laboratories, CMELS, 5000 East Ave. Livermore, CA 94551. \\ **Harvard University, Cambridge, MA, 02138.
}

\begin{abstract}
Ion beams of sufficient energy to erode a surface can lead to surface modulations that depend on the ion beam, the material surface it impinges, and extrinsic parameters such as temperature and geometric boundary conditions. Focused Ion Beam technology both enables site-specific placement of these modulations and expedites research through fast, high dose and small efficient use of material. The DualBeam (FIB/SEM) enables in situ metrology, with movies observing ripple formation, wave motion, and the influence of line defects. Nanostructures (ripples of $>400 \mathrm{~nm}$ wavelength to dots spaced $<40 \mathrm{~nm}$ ) naturally grow from atomically flat surfaces during erosion, however, a steady state size may or may not be achieved as a consequence of numerous controlled parameters: temperature, angle, energy, crystallography. Geometric factors, which can be easily invoked using a FIB, enable a controlled component of deposition (and/or redeposition) to occur during erosion, and conversely allow a component of etching to be incurred during (ion-beam assisted) deposition. High angles of ion beam inclination commonly lead to "rougher" surfaces, however, the extreme case of $90.0^{\circ}$ etching enables deposition of organized structures 1000 times smaller than the aforementioned, video-recorded nanostructures. Orientation and position of these picostructures (naturally quantized by their atomic spacings) may be controlled by the same parameters as for nanostructures (e.g. ion inclination and imposed boundary conditions, which are flexibly regulated by FIB). Judicious control of angles during FIB-CVD growth stimulates erosion with directionality that produces surface modulations akin to those observed for sputtering. Just as a diamond surface roughens from 1-D ripples to 2-D steps with increasing angle of ion sputtering, so do ripples and steps appear on carbon-grown surfaces with increase in angle of FIB-CVD. Ion beam processing has been a stalwart of the microelectronics industry, is now a vital tool for research of self-organizing nanostructures, and promises to be a focus for future picotechnology.
\end{abstract}

\section{INTRODUCTION}

An ion beam impinging a surface, in addition to eroding away material, supplies energy that enables a self-organizing morphology to develop [1,2]. Modulations can depend on many parameters [2-10]: intrinsic to the ion beam (eV, angle, species, dose); intrinsic to the sample (species, crystallography, grain orientations, boundary conditions such as pre-patterned 3-dimensional geometries [4-6] or interfaces and crater sidewalls [7-8,11]); and/or extrinsic (temperature, chemical enhancers). Ripples can develop independent of whether a big static ion beam or a Focused Ion Beam is used [3-7]. The scan direction of the FIB can be irrelevant to the ripple orientation [4-9], with a caveat that the scan pattern is a wellcontrolled, overlapping-digital pattern. Even boundary conditions can be the same for FIB or static beams [4-6], however FIB generally creates 3-D geometries, and boundary conditions such as aspect ratio are paramount to FIB processing [7-8]. As we etch deeper, the yield drops. Material cannot get out of the hole and redeposition has been measured as a source term to enable ripple growth [7-8]. FIB also enables fast, high doses while consuming minimal resources, thus FIB efficiently prototypes research that may then port back to static beam processing.

Ripples grow. But what controls steady state? What stops ripple growth? Starting from an atomically flat surface such as single crystal Si or diamond, ripples must grow. Ripples on diamond grow, depend on 
angle [9], and have been reported to saturate [10]. Redeposition can help ripples grow [8]. Redeposition increases with the aspect ratio of a micronscale hole, and this effect increases when the ion beam impinges at an angle [7]. In between each ripple is similarly considered a nanoscale hole. Thus atoms trying to escape from within the ripples become redeposited as a consequence of the aspect ratio of the ripple itself. Redeposition is traditionally nondesirable (bad), but it can be controlled either to enhance growth and ripple uniformity or to self-organize millions of picostructures.

\section{EXPERIMENTAL PROCEDURES \& RESULTS}

Surfaces are energized by ion sputter etching with a FIB (model FEI-NOVA600-DualBeam) with 30keV $\mathrm{Ga}^{+}$ions; and all imaging is performed using an SEM operated at $5 \mathrm{keV}$ and variable currents. Carbonbased materials are studied: single crystal diamond, CVD-carbon-coatings on SiC fibers, and FIB-CVD carbon. Additional processing parameters are listed in $[7,8,11,13]$.

An in situ movie is acquired of the developing surface morphology, with the SEM imaging at the same time as the FIB etches $(5 \mathrm{nA} @ 30 \mathrm{keV})$. Both beams are rastered but deliberately out of sync to minimize interference noise (e.g. ignore straight horizontal lines). Furthermore, FIB produces secondary electrons, thus the current of the SEM $(1.6 \mathrm{nA} @ 5 \mathrm{keV})$ is greater than normal so that the image corresponds to where the SEM is rastering [11]. A third consideration is that the SEM beam is rastered only partially on the area of FIBing. Observing the edge of the crater provides a constant frame of reference. For this movie the ion angle of incidence is $60^{\circ}$, and the SEM is imaging nearly normal to the surface. During a 57 minute movie acquisition, the FIB etching parameters are: a total dose of $33 \times 10^{18}$ ions $/ \mathrm{cm}^{2}$, a total etch depth of $6400 \mathrm{~nm}$, and a final aspect ratio of 0.23 depth/length (width is treated as infinite [7]). Yield (atoms off per ion in) drops throughout with the final average yield $=3.4$. Reference yields for this angle are 4 [9], but quantifying yield vs. aspect indicates the early stage (aspect $<0.1$ ) has yield as high as 5 [7].
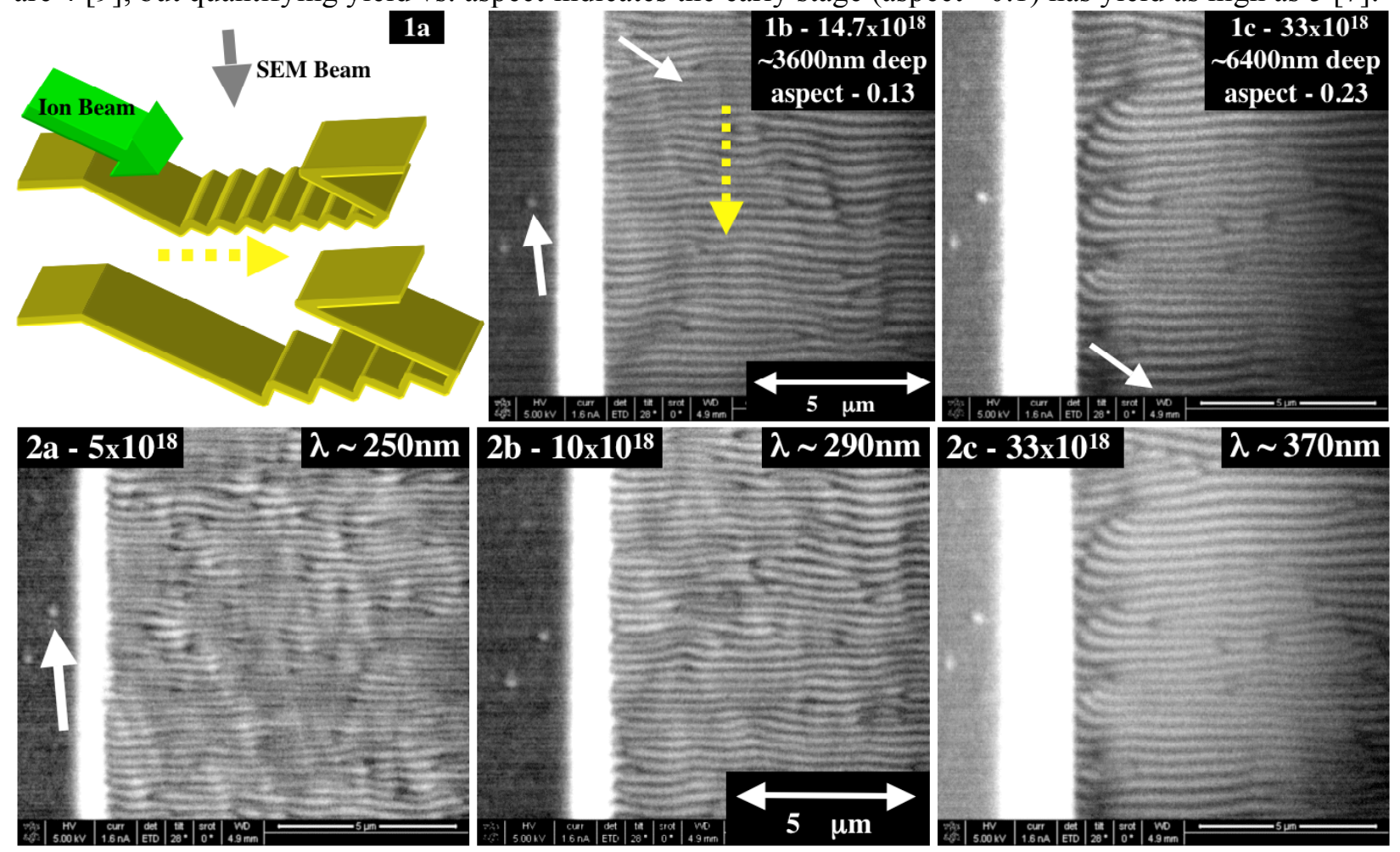

Figure 1: Clips from SEM movie of FIB etching of single crystal diamond with an ion beam inclination of $60^{\circ}$ from the north. Movie shows wave motion southward. (Arrows point to same wave.) However, schematic (rotated $-90^{\circ}$, aspect exaggerated) shows motion is mostly the process surface moving downstream. Wave motion appears nearly linear with dose: $\sim 1$ micron per $4.8 \times 10^{18}$ ions $/ \mathrm{cm}^{2}$. Yield drops as aspect ratio of crater increases [7]; avg. yield =4.3 until Fig.1b, but avg. yield drops to 2.7 between Fig. 1b and 1c. Clips of Figure 2 show ripples develop and grow as a function of fluence, depth, aspect ratio (0.23 in Fig. $2 \mathrm{c})$ of the etch pit, and redeposition [7-11]. SEM imaging is $8^{\circ}$ from normal which modified y measurements by $1 \%$. Arrow aligns imaging drift to a defect left of crater. 
Ripple waves move, but how fast? The movie shows the ripples moving southward, akin to waves pushed downwind. Figure 1 presents two SEM clips with arrows indicating the same wave that has propagated southward. However, the ion beam inclination causes the entire processed surface to move southward. What appears as a motion of 8.7 microns from Fig. $1 \mathrm{~b}$ to $1 \mathrm{c}$ is actually only 3.8 microns.

Ripples grow, but how much? Clips in Figure 2 at doses of 5,10 , and $33 \times 10^{18}$ ions $/ \mathrm{cm}^{2}$, have wavelength increasing from 250 to 290 to $370 \mathrm{~nm}$, respectively. These wavelengths all exceed the $\sim 220 \mathrm{~nm}$ saturation reported [10] at a dose of only $1-2 \times 10^{18}$ ions $/ \mathrm{cm}^{2}$. Wavelength correlates better to aspect than to dose [8].

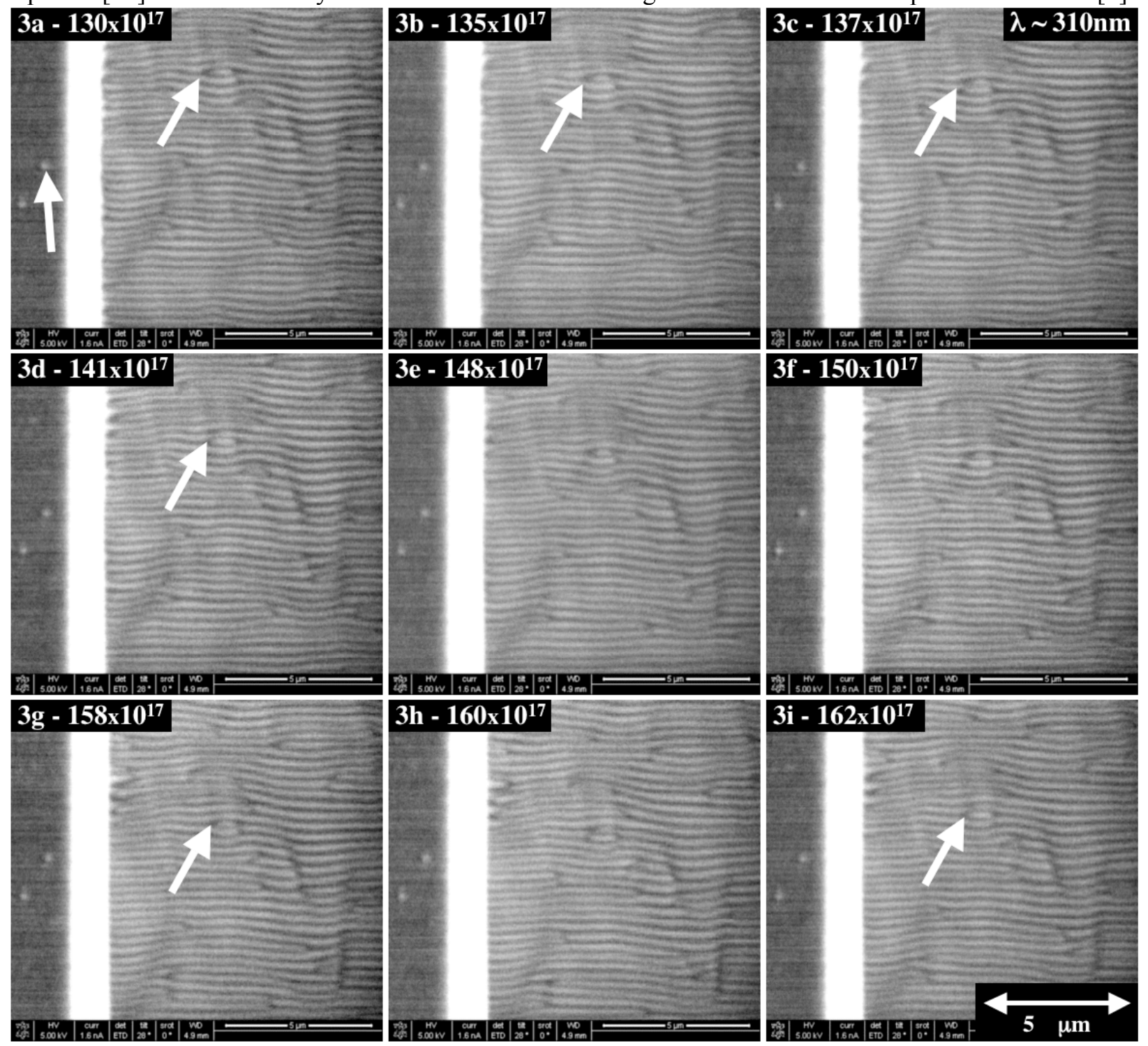

Figure 3: Clips from SEM movie show defects move thrice the speed of surrounding waves. Defects come and go, however, as ripples grow larger the defect density drops. Wave defects do not always exhibit energetically logical line defect reactions. An extrinsic fault line (Fig3a) shrinks, but rather than annihilating itself, it breaks neighboring line to jump forward in Figs. $3 \mathrm{~b}$ and 3c, and regrows in length in Fig3d. The process repeats to Fig. 3g, and then again to Fig. 3i. (Modeling wave motion via redeposition downstream helps rationalize this defect motion.)

Ripple defects move faster, three times as fast, but why? Figure 3 presents clips monitoring an extrinsic line fault skipping ahead 3 waves as dose increases from 13 to $16.2 \times 10^{18}$ ions $/ \mathrm{cm}^{2}$. The extrinsic fault jumps ahead by sequential breaking and joining lines, akin to dislocation and stacking fault motion. Although the extrinsic fault shrinks before each jump, it resists self-annihilation as is the energetic solution in dislocation dynamics. Figure 4 presents two opposing line defects in close proximity, which 
refuse to annihilate. From classic line-tension theory, the energy of the two opposing dislocations would make them annihilate, by having the trailing right edge jump first. If some thing ("force") encourages the left to jump ahead first, then the left should try to continue leaving the right behind. However, a model of redeposition has it increasing just downstream of the ends of the defects as they more efficiently erode. Thus the redeposition builds the downstream defect as it erodes the upstream one.

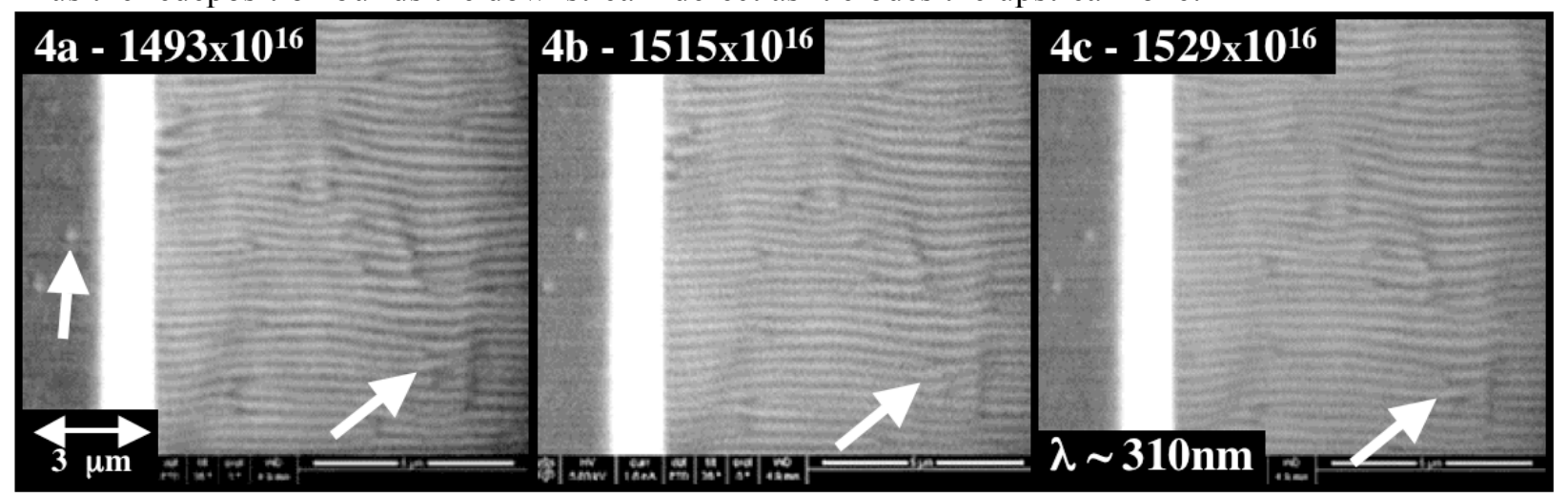

Figure 4: Clips from SEM movie showing two opposite edge defects in close proximity, a left edge ahead of a right edge and separated by a single full line (Fig. 4a). As the waves propagate, the left edge jumps ahead and the two "dislocations" become separated by two full lines (Fig. 4b). And then the right edge jumps ahead to return the configuration condition to only one full line between the edges. (And this repeats.)

Ripples grow, but can we grow them smaller $[8,11,12]$ ? Figure 5 considers the case of FIB ion etching for TEM sample preparation. With redeposition a source term in ripple development [7,8], we also consider that the ion etch process includes contamination of surfaces with the impinging ions, and both of these are considered TEM sample preparation artifacts that are to be minimized. (I.e. a few atoms are always deposited, but usually many more are removed.) A second consideration is that modulations often show an increase in amplitude with increasing angle [2,9]; however, a TEM prep condition offers the most ultimate $90.0^{\circ}$ angle (pure grazing), while also requiring atomically flat surfaces as a final condition $[8,11]$. And thirdly, the final FIB polishing steps for TEM prep are reaching a condition where the etch rate (due to geometry and precision) is intentionally dropping to 0 (Fig. $5 \mathrm{~d}$ ). Although ideal aspect $=0$ for atomically flat prepared surfaces will mean redeposition can be minimal, suddenly deposition itself is an actuality. At the last instance, our topmost layer of the sample will be the most heavily contaminated with the depositing ion species. (For a Ga-FIB, such Ga atoms do not have to subsequently vaporize off the surface.) Yet the energy of the ion beam still self-organizes the surface at the picoscale.

Ripples grow, but can we grow them up instead of down? Truly, ion erosion ripples are growing down, down below the original surface (Fig. 1a); and especially in the case of FIB, the production of selforganized structures at the bottom of a pit may have limited utility (although imprinting may reverse them to the top of a pedestal) $[6,8]$. If we can control deposition during erosion to grow picostructures, can we control erosion during deposition? As carbon pads are FIB-CVD grown in Figure 6, the modulations forming on surfaces depend on angles akin to how erosion angles influence 1D ripples vs. 2-D steps. Modulation wavelength also increases from $230 \mathrm{~nm}$ to $340 \mathrm{~nm}$ with increased angle for growing ripples, similar to erosion ripples. However, modulation also depends on many growth parameters (current, pressure and scan rate $[11,12]$ ) and modulations also appear to extend below the top surface. Carbon pad growth is viable by the SEM (EBAD - electron beam assisted deposition), too. Thus 2-beam processing [6] can have the ion beam angle, current, site-specificity, etc. all independent of the growth.

\section{DISCUSSION}

Ripples grow, and a movie watches them grow. Ripples grow due to energy from the ion beam, but the movie adds energy of the electron beam, too [15]. The impinging FIB generates secondary electrons, thus the SEM movie has to invoke a sufficiently high current of incident electrons to ensure SEM-produced secondary signal is dominant. SEM energy modifies both ripples and yield. When we "see" ripples form, the electrons "do" further energizing of the ripples [15]. If we watch the FIB etch a crater, the crater ends 
up less deep. Yield drops, redeposition increases, ripples grow. In situ SEM can enhance redeposition (Ebeam-CVD [6]) and reduce overall etch rate. This drop decreases aspect ratio, which should reduce ripple wavelength [7]. If SEM energy only enhanced surface mobility, a reduction of surface tension should reduce ripple growth. However, the enhanced redeposition by EBAD provides ripple growth.
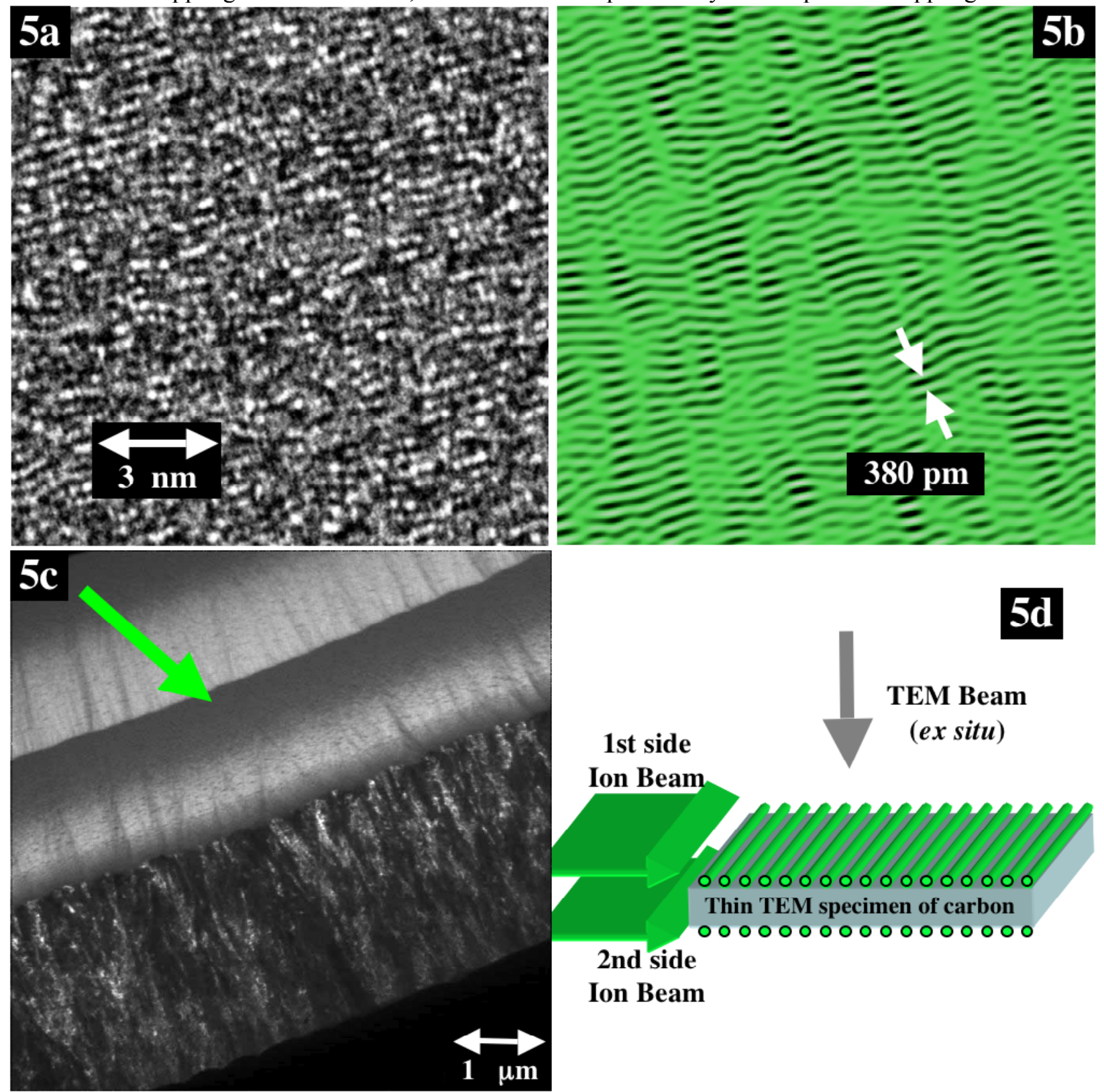

$5 d$
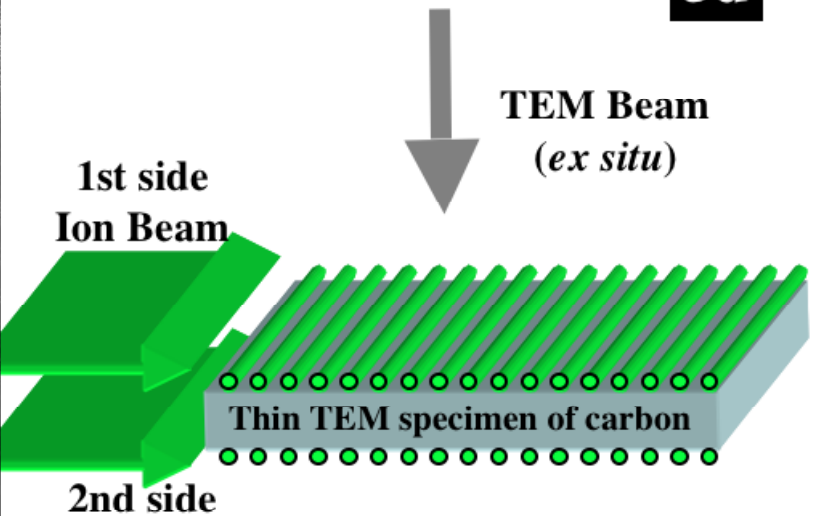

Ion Beam

Figure 5: FIB preparation of TEM section of coatings on SiC fibers [13] provides an ion beam incidence of $90.0^{\circ}$ ! Ripples would like to grow, but FIB polishing attempts to keep TEM sample surfaces atomically flat. Ion induced contamination is relegated to the topmost layer. Yield should be high at this angle, but TEM prep drops erosion rate to zero as the last step. Redeposition becomes deposition of the ion beam itself, yet the beam energy for selforganization persists. Surface modulations 1000 times smaller than the nanostructures of earlier figures arguably are defined picostructures or $1-\&-1 / 2-\mathrm{D}$ crystals, quantized perfectly to the natural $\mathrm{Ga}$ atomic spacing. Image processing by fft-filtering (Fig. $5 b$ ) removes the majority of the carbon TEM specimen from the TEM image. Dark Field imaging (Fig. 5c) shows ripples are ordered over tens of millions, but also that the ripples are relegated only to carbon-based surfaces and not polycrystalline $\mathrm{SiC}[11,13]$, and ripples prefer to align with boundaries within the multilayers rather than normal to ion beam angle rotated $30^{\circ}$ to boundary (green arrow) $[4,8]$. 
Ripples and sputter yields are interdependent, and both depend on aspect ratio. Yield is often listed as a constant, but it increases as ripples initially form [1], and then ripple growth in turn causes yield to decay [14]. Redeposition diminishes yield but can cause ripples to continue growing [8]. Redeposition can be further increased at high aspect due to excess chemical enhancers or even the electron beam used for in situ movies. Electron and ion beams create local charge fields, which may influence redeposition of sputtered atoms, especially non-neutral species. However, steady state is achieved and monitored by lack of sample drift during imaging. Whereas ex situ FIB etching at $60^{\circ}$ provided average yields $>4.5$ up to aspect $>0.5[7,8]$, the additional SEM in situ caused the average yield to drop to 4.3 at aspect $=0.13$ and to 3.4 at aspect $=0.23$. Datta, et al [10] observed wavelength saturation at $220 \mathrm{~nm}$ at $\operatorname{dose}<2 \times 10^{18}$ ions $/ \mathrm{cm}^{2}$. However, while increasing dose to $>35 \times 10^{18}[7]$, the aspect was increased (and adjusted from 0.1 to 0.8 ), which caused the yield to drop and caused ripple wavelength to increase to nearly 500nm [8]. By adding electron beam energy during an in situ movie, the wavelength can be increased to $\sim 300 \mathrm{~nm}$ at an aspect of $\sim 0.13$, whereas without movie a wavelength of $\sim 300 \mathrm{~nm}$ needed an aspect $>0.2$.
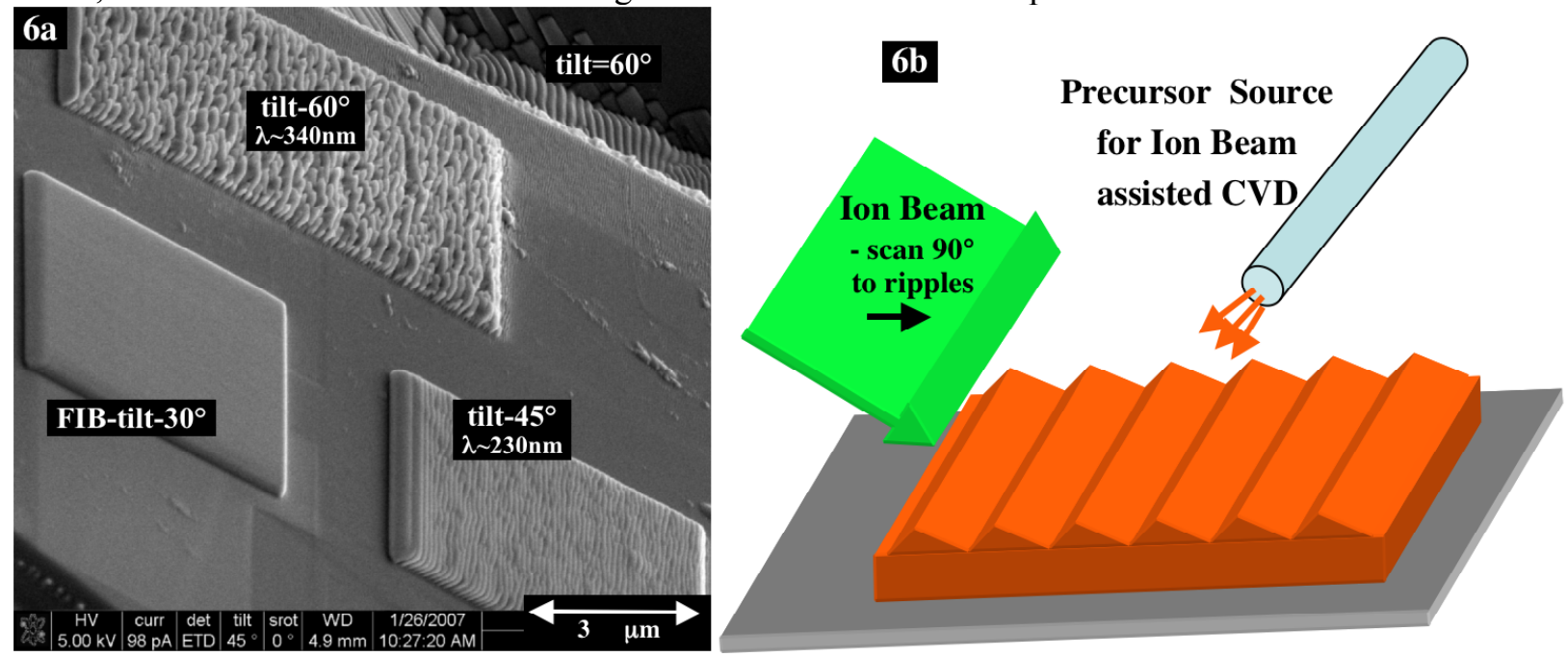

Figure 6: (a) SEM image of carbon pads grown by FIB-CVD, with ion beam at inclinations of: $30^{\circ}$ tilt to produce a smooth surface, $45^{\circ}$ a set of 1-D ripples, and $60^{\circ}$ 2-dimensional steps (akin to erosion angles [9,11,12]). FIB scan is intentionally normal to ripples (i.e. ripples relate to inclination angle and are not caused by FIB scanning.) Ripples grown on top of pads have more engineering utilities than erosion ripples at bottom of pit (in background of Fig. 6a). 2-beam processing adds flexibility with the SEM for EBAD and FIB only for site-specific erosion modulations [6].

\section{ACKNOWLEDGMENTS}

This work was performed under the auspices of the United States Department of Energy by the Lawrence Livermore National Laboratories under contract of No. DE-AC52-07NA27344. UCRL-PROC-236448.

\section{REFERENCES}

1. P. Sigmund, J. Materials Science 8 (1973) 1545-1553.

2. R.M. Bradley and J.M.E. Harper, J. Vac. Sci. Technol. A 6 (1988) 2390-2395.

3. E. Chason, et al, Appl Phys Rev. 81(10) (1997) 6513-6561.

4. A. Cuenat and M.J. Aziz, Mater. Res. Soc. Symp. Proc. 696 (2002) p. N2.8.

5. S. Ichim and M.J. Aziz, J. Vac. Sci. Technol. B 23 (2005) 1068-1071.

6. W.J. MoberlyChan, D.P. Adams, M.J. Aziz, G. Hobler, T. Schenkel, MRS Bull., 32 (2007) 424-432.

7. W.J. MoberlyChan, T.E. Felter, and M.A. Wall, Microscopy Today, Nov. (2006) 28-34.

8. W.J. MoberlyChan, Mater. Res. Soc. Symp. Proc. 960 (2006) p. N10-02.

9. D.P. Adams, M.J. Vasile, T.M. Mayer, \& V.C. Hodges, J. Vac. Sci. Technol. B 21 (2003) 2334-2343.

10. A. Datta, Y.R. Wu, and Y.L. Wang, Phys. Rev. B 63 (2001) 125407.

11. W.J. MoberlyChan, Microsc. Microanal. 13(Supp 2) (2007) 598-599.

12. W.J. MoberlyChan et al, submitted to Nature.

13. J.V. Marzik, W.J. Croft, R.J. Staples, W.J. MoberlyChan, Mater. Res. Soc. Symp. Proc. 982 (2006).

14. G. Carter, J. Appl. Phys. 85(1) (1999) 455-459.

15. W.J. MoberlyChan, Mater. Res. Soc. Symp. Proc. submitted (2008). 